\title{
Polluting Productions and Sustainable Economic Growth: A Local Stability Analysis
}

\author{
Giovanni Bella ${ }^{1}$ \\ ${ }^{1}$ University of Cagliari, Italy. \\ Email: bella@unica.it \\ Received January $25^{\text {th }}, 2009$; revised February $24^{\text {th }}, 2009$; accepted March $10^{\text {th }}, 2009$.
}

\begin{abstract}
The aim of this paper is to analyze the link between natural capital and economic growth, in a Romer-type economy characterized by dirty emissions in the production process, and to examine the conditions under which a sustainable growth, which implies a decreasing level of dirty emissions, might be both feasible and optimal. This work is close to Aghion-Howitt (1998) with some more general specifications, in particular regarding the structure of preferences and the technological sector. We also deeply study the transitional dynamics of this economy towards the steady state, and conclude that a determinate saddle path sustainable equilibrium can be reached even in presence of a long run positive level of polluting emissions, thanks to a growing level of new home-made inventories, without whom some indeterminacy problems are likely to emerge.
\end{abstract}

Keywords: local stability, sustainable growth, aghion-howitt model

\section{Introduction}

It is commonly believed that economic development might lead to overexploitation of natural resources and intensification of environmental damages, as for example the augment of carbon dioxide concentrations in the atmosphere due to an increase in transportation services. On the contrary, empirical evidence suggests that rich societies seek a less polluted environment to live in, so they are more willing to invest in abatement technologies and enforce environmental regulations. The logical consequence must be that economic activity will then also lower the dirtiness of any existing production technique, which leaves the door open, for example, to those supporting the so-called Environmental Kuznets Curve hypothesis [1].

During the last three decades, this counterposition encouraged many economists to develop models in which economic growth depends on the extractive use of the environment. Inspired by the work of the Club of Rome and its pessimistic view on the possibility to attain longrun growth under environmental constraints, these models tried to depict the conflict between growth and the environment. Over time the variety of models grew rapidly, differing not only with respect to the basic framework adopted but also with respect to the type of environmental resource being considered and the problem analyzed, mainly because each model has specific properties that become useful for the analysis of either specific economic concerns.

More recently, research on endogenous growth and the environment turned more and more attention from one- to multi-sector models, where knowledge accumulation might have the potential of lowering environmental damages through an increase in technological progress $[2,3,4,5]$. Likewise, in the seminal paper of Aghion and Howitt [6], to whom we will be referring to as $A H$ from now on, it is shown that an unlimited growth can indeed be sustained when account is taken of both environmental resource use and innovation in abatement activities [7,8].

Broadly speaking, the properties of endogenous technical change have been widely investigated in the existing economic literature, with some indeterminacy problems and Hopf bifurcating outcomes being of particular concern [9]. On the contrary, we want to show in this paper that the introduction of the environmental issue can drive the economy back to a unique, locally stable, equilibrium solution, where sustainability of consumption is finally reached. However, this occurs only if a specific sustainability rule, stating that consumption and natural capital grow at the same rate, is to be followed, which is also consistent with a forward looking individual behavior and no myopic statement of the adopted social policy. Therefore, the basic question we want to address is whether a sustainable path can be reached even if some dirty production processes, assumed here to be necessary for any economic activity are adopted.

To this bulk of literature this paper devotes particular attention, aimed at developing a model close to $A H$, that considers pollution as a choice variable entering the production function as a measure of dirtiness, whose exter- 
nal effects allow to increase the level of output [10]. It seems then to be interpreted as pollution is a necessary part of production and economic growth. Moving a step forward from $A H$, we also deeply concentrate on the study of the transitional dynamics of the model, and provide the whole necessary and sufficient conditions for the existence of a feasible steady- state equilibrium path associated with a positive long-run growth. Moreover, we conclude that a determinate saddle path sustainable equilibrium can be reached even in presence of a long run positive level of polluting emissions, thanks to a growing level of new home-made inventories, without whom some indeterminacy problems are likely to emerge $[11,12]$.

The rest of the paper is organized as follows. In section 2 , we derive the formal structure of the model, with particular attention to the set of preferences, the level of technology, and the introduction of pollution as a crucial variable for the system to grow. In Section 3, we concentrate on the solution of the optimization problem, and deeply investigate the stability properties of the associated steady state solution. The transitional dynamics of this economy will provide some interesting results and policy suggestions on the way to drive an economy along a sustainable growth path. A final section concludes, and a subsequent Appendix provides all the necessary proofs.

\section{A Model with Dirtiness}

Before we enter the algebraic version of the model, we ought to provide some detailed explanations related to the production function, the dynamics of the environment, and the set of preferences used to characterize the economy, whose properties we want to investigate in the rest of the paper.

Following Romer, 1990, let $S_{0}$ represent the fixed amount of skilled labour, which can be devoted to production of the final good, $S_{Y}$, or to improvement of technology, $S_{A}$. Henceforth, we will normalize the problem by assuming

$$
S_{0}=S_{Y}+S_{A}=1
$$

In particular, technology $(A)$ is not fixed. It can be created by engaging human capital in research, growing over time according to

\footnotetext{
${ }^{1}$ It is assumed that technology does not depreciate

${ }^{2}$ Remember that in Romer, 1990, technology is assumed to be made up of an infinite set of designs for capital, which (for simplicity) enter the production function in an additively separable manner, given by

$$
Y=\eta^{\alpha+\beta-1}\left(S_{Y} A\right)^{\alpha}(L A)^{\beta} K^{1-\alpha-\beta} \quad 0<\alpha, \beta<1
$$

where $\eta$ represents the units of capital goods to produce one unit of any type of design. Here we assume, for simplicity, that there is no unskilled labour; that is all workers are supposed to be specialized. Let us then consider $L=S_{Y}$ to derive Equation (3), and normalize the scale parameter to unity, for simplicity $\left(\eta^{\alpha+\beta-1}=1\right)$.

${ }^{3}$ This production function exhibits constant returns to scale at a disaggregate level because each firm takes $z$ as given. On the contrary, a social planner can internalize this kind of externality, due to pollution intensity, thus obtaining increasing returns.
}

$$
\frac{\dot{A}}{A}=\varphi+\not S_{A}
$$

$\gamma$ indicates the research success parameter. Let us then assume that technology $A$ be partly the result of endogenous (home-made) R\&D efforts, $\gamma S_{A}$, whilst the remaining part depends on some exogenous new inventories, whose spill-over effects can be synthesized through a constant catch-up parameter, $\varphi$ [13]. We assume $\dot{A} / A=\varphi+\gamma S_{A}>0$, as long as either $\varphi$ or $\gamma$ and $S_{A}$ are set positive. ${ }^{1}$ Thus, technology can grow without bound. We will show afterwards that, if we relax the positiveness assumption on $\gamma$, the economy will face the emergence of some undesired and indeterminate equilibrium problems.

Moreover, although technology is not directly linked to pollution here, we basically consider the discovery of new goods, or new (i.e. less polluting) production processes, as the implicit way societies follow to broadly reduce their dependence from environmental resources. Basically, we are saying that each new inventory due to technological advance is also assumed to be cleaner than the previous one. This is also consistent with the empirical evidence that developed societies seek a less polluted environment to live in.

Note also that research activity is assumed to be human-capital-intensive and technology-intensive, with no capital $(K)$ and ordinary unskilled labour $(L)$ engaged in that activity. To produce the final good $Y$, however, $K$ does enter as an input along with human capital $S_{Y}$ and technology $A .^{2}$ According to the assumptions made in $A H$, the main feature of this economy is that production is also affected by another variable indicating the intensity of pollution, $z(t) \in[0,1]$, such that higher values of $z$ yield more of the good but also more pollution ${ }^{3}$

$$
Y=A^{\alpha}\left(1-S_{A}\right)^{\alpha} K^{1-\alpha} z
$$

We may also consider $z$ as a measure of dirtiness of the existing production technique [10]. For example, focus on cheese manufacturing. Only a fraction of the raw milk processed gives rise to white cheese (or other diary products), the remaining is called whey, a liquid by-product, only partially recyclable, which constitutes the greater part of the resulting pollution loads. In other words, we are assuming that production of output arises at the expenses of the environment, with some polluting emissions being necessarily needed.

Moreover, it is assumed that the flow of pollution $P$ is proportional to the level of production, and that the use of cleaner technologies (which means low values of $z$ ) reduces the pollution/output ratio. ${ }^{4}$ Formally,

$$
P=Y z^{\gamma}
$$

${ }^{4}$ The extractive use of the environment in production can either be modeled as an input to production or, like here, as a by-product of production; that is, pollution influences output indirectly. 
To clarify the utility of using both variables, $P$ and $z$, as two sides of the same coin (the damages to the environment), let us make another example that can be drawn from current industrially advanced economies. Basically, oil combustion is being needed either to feed the engine of our cars or to stoke the furnaces of our firms, with $\mathrm{CO}_{2}$ emissions being an unavoidable consequence. Referring to our model it would imply that only a fraction of the oil burnt $(z)$ serves to produce final output $(Y)$, the rest being pushed into the atmosphere as a resulting emissions' burden. Nevertheless, it is indeed true that not all of these emissions are damaging, since carbon sequestration due to forests allows, for example, to reduce the total pollution loads.

What distinguishes this economy from the one defined in $A H$ is that we let pollution, $P$, depend also on the parameter expressing research success in technological advances, $\gamma$; that is like assuming that the bigger $\gamma$-values the smaller the impact of dirty techniques on pollution, and then the cleaner the ecosystem. ${ }^{5}$

On the other hand, the level of investment in physical capital is given by the usual functional form $\dot{K}=Y-C$.

\subsection{Dynamics of the Environment}

Commonly, the environmental sector can be represented by the dynamics of the stock of natural capital available to the economy, $E$ :

$$
\dot{E}=N(E)-P
$$

where $N(E)$ determines the speed at which nature regenerates, while $P$ measures the negative effect due to polluting emission. ${ }^{6}$ The former is constantly reduced not only by economic activities, but also by non-anthropogenic processes, such that ecosystems have to devote part of their regeneration capacity to the maintenance of their own structure. ${ }^{7}$

If the capacity for regeneration exceeds the requirements for maintenance, $N(E)$ becomes positive. $N(E)$ can therefore be interpreted as the difference between natural resource reproduction and resource use for maintenance [14] that determines nature's capacity to recover from pollution and resource extraction $[4,5]$.

Some authors [15] propose a linear representation of the regeneration function

$$
N(E)=\theta E
$$

\footnotetext{
${ }^{5}$ Conversely, a negative value of $\gamma$ reduces abatement programs, thus finally increasing the amount of pollution realized.

${ }^{6} \mathrm{We}$ follow here the broad definition of natural capital given by Costanza and Daly, 1992.

${ }^{7}$ Conventional wisdom holds that plants will purify the air, helping to reduce concentration of harmful gases. But, recently, it has been shown that when temperatures exceed a threshold, trees and other plants emit chemicals that encourage toxic ozone production (Science, 2004).

${ }^{8}$ Although several criticisms have been raised against the algebraic simplicity of this specification (e.g., Rosendahl, 1996), it remains still widely used in the literature of the field, as for example in our reference model of Aghion and Howitt, 1998.
}

where $\theta$ denotes the constant rate of regeneration. ${ }^{8}$

Following this approach, if we substitute both Equations (4) and (6) into (5), we explicitly end up with

$$
\dot{E}=\theta E-Y z^{\gamma}
$$

which represents the environmental constraint to be used in the subsequent maximization problem.

\subsection{The Set of Preferences}

Let the preferences of the representative agent depend either on the level of consumption, $C_{t}$, or the stock of natural capital available to the economy, $E_{t} \cdot{ }^{9}$ The intertemporal utility function is then given by

$$
\int_{0}^{\infty} U\left(C_{t}, E_{t}\right) e^{-\rho t} d t
$$

where $\rho$ is the social discount rate. ${ }^{10}$

$U(\cdot)$ is continuous, twice differentiable, and possesses the following properties: $U_{C}>0, U_{E}>0, U_{C C} \leq 0$. Also suppose that $U(\cdot)$ is concave with respect to its two arguments: $U_{C C} \cdot U_{E E}-\left(U_{C E}\right)^{2} \geq 0 .^{11}$

Theoretically, sustainable development usually comprises two conditions. Firstly, a non-decreasing level of consumption or utility levels, and secondly a constant or improving state of the environment. Whether sustainable development in this sense can be optimal, depends on the functional form of the utility function [4]. ${ }^{12}$

A specific utility function is assumed here to have the following CES structure

$$
U(\cdot)=\frac{(C E)^{1-\sigma}-1}{1-\sigma}
$$

where $\sigma>0$ represents the inverse of the intertemporal elasticity of substitution.

This functional form guarantees that both $C$ and $E$ grow at the same rate, so that the $C / E$ ratio is constant in equilibrium [16]. ${ }^{13} \mathrm{We}$ show in the next

${ }^{9}$ One interpretation would be forests, which contribute to welfare both as sources of timber and also as stocks which provide many ecosystem services to society (for example, carbon's sequestration, preservation of bio-diversity)

${ }^{10}$ For simplicity, time subscripts will be omitted in the rest of the paper ${ }^{11}$ Constraints to the optimization problem could, for example, be introduced by defining critical minimum levels for natural capital (Barbier and Markandya, 1990) or by excluding decreasing utility paths (Pezzey, 1992). But as these restrictions usually involve inequality constraints, they may complicate the optimization problem considerably

${ }^{12}$ While $A H$ deal (to simplify the analysis) with a logarithmic, thus separable, utility function, we prefer to introduce a non-separable function instead (as in Musu, 1995), that allows to compare consumption and environmental quality as two substitutes, according to agents' tastes towards them. Nonetheless, it will be shown that both assumptions can be finally reconciled

${ }^{13}$ We show that an improvement in natural capital is conductive to growth only if we assume that consumption and natural capital are substitutes, which implies $U_{C E}<0$. Therefore, households will be willing to postpone part of their consumption opportunities only if the expected stock of natural capital is improved 
section that this assumption is rich of powerful consequences. In particular, for growth to be balanced, it will allow us to both derive (in equilibrium) a constant lower-bound level of dirty emissions, and a constant level of the pollution/output ratio either.

\section{The Social Planner Maximization Problem}

We assume that the social planner has to maximize the following discounted CES utility function,

$$
\int_{0}^{\infty} \frac{(C E)^{1-\sigma}-1}{1-\sigma} e^{-\rho t} d t
$$

subject to the following constraints:

$$
\begin{aligned}
\dot{K} & =A^{\alpha}\left(1-S_{A}\right)^{\alpha} K^{1-\alpha} z-C \\
\dot{A} & =\left(\varphi+\gamma S_{A}\right) A \\
\dot{E} & =\theta E-A^{\alpha}\left(1-S_{A}\right)^{\alpha} K^{1-\alpha} z^{1+\gamma}
\end{aligned}
$$

and given initial positive values:

$$
A(0)=A_{0} \quad K(0)=K_{0} \quad E(0)=E_{0}
$$

The current value Hamiltonian is given by

$$
\begin{gathered}
H_{c}=\frac{(C E)^{1-\sigma}-1}{1-\sigma}+\lambda\left[A^{\alpha}\left(1-S_{A}\right)^{\alpha} K^{1-\alpha} z-C\right]+ \\
+\mu\left[\theta E-A^{\alpha}\left(1-S_{A}\right)^{\alpha} K^{1-\alpha} z^{1+\gamma}\right]+\vartheta\left[\left(\varphi+\gamma S_{A}\right) A\right]
\end{gathered}
$$

where $\lambda, \mu$ and $\vartheta$ denote the costate variables associated with the accumulation of physical capital, natural capital and knowledge capital, respectively. ${ }^{14}$

Solution to this optimal control problem implies the following necessary first order conditions ${ }^{15}$

$$
\begin{gathered}
C^{-\sigma} E^{1-\sigma}=\lambda \\
\lambda=\mu(1+\gamma) z^{\gamma} \\
\lambda \alpha A^{\alpha}\left(1-S_{A}\right)^{\alpha-1} K^{1-\alpha} z-\mu \alpha A^{\alpha}\left(1-S_{A}\right)^{\alpha-1} K^{1-\alpha} z^{1+\gamma}=\vartheta \gamma A
\end{gathered}
$$

accompanied by the equation of motion for each costate variable, that can be obtained with a bit of mathematical manipulation:

$$
\begin{aligned}
& \frac{\dot{\lambda}}{\lambda}=-\left(\frac{\gamma}{1+\gamma}\right)(1-\alpha) A^{\alpha}\left(1-S_{A}\right)^{\alpha} K^{-\alpha} z+\rho \\
& \frac{\dot{\mu}}{\mu}=\rho-\theta-\frac{C}{E}(1+\gamma) z^{\gamma} \\
& \frac{\dot{\vartheta}}{\vartheta}=\rho-(\varphi+\gamma)
\end{aligned}
$$

and the transversality conditions for a free terminal state, whose specification is provided in the Appendix, that jointly constitute the so-called canonical system.

Questions of interest include: how does pollution affect

\footnotetext{
${ }^{14}$ Appendix A derives the optimality conditions, which will be discussed in the rest of this section

${ }^{15}$ Necessary condition for a maximum can be checked by studying the sign of all principal minors of the Hessian matrix for the control variables of the problem, whose determinant is formed by the following signs
}

the growth rate of this economy in the steady state? And particularly, what is the optimal level of dirtiness? The basic feature of such a steady state implies that:

Remark 1 Along a sustainable balanced growth path (BGP):

1) The marginal rate of substitution between $C$ and $E$ is constant, $M R S_{C, E}=\varepsilon<0$.

2) Both $C$ and $E$ grow at a constant rate, $g=\frac{\rho-(\gamma+\varphi)}{1-2 \sigma}$.

3) The degree of dirtiness, $z$, is constant.

4) The BGP is non-degenerate and the growth rate of the economy is positive.

In particular, from FOC's we can easily derive the following Bernoulli's differential equation for $z$,

$$
\gamma \dot{z}+\phi z=\varepsilon(1+\gamma) z^{1+\gamma}
$$

where $\phi=\gamma+\varphi-\theta$. More interestingly, a stable steady state occurs when

Remark 2 The rate of new technological advances is lower than the speed at which nature regenerates $(\phi<0)$, and the level of dirty emissions converges to a positive minimum threshold, $\tilde{z}$ (stable equilibrium).

If we concentrate on the stable solution, evolutionary path for $z(t)$ follows consequently.

As depicted in Figure 1, when approaching the steady state the level of dirtiness $(z)$ lowers, but never collapses to zero, $\tilde{z}=\left[\frac{\phi}{\varepsilon(1+\gamma)}\right]^{\frac{1}{\gamma}}$. It can be interpreted as an economy that moves along a long run sustainable path thanks

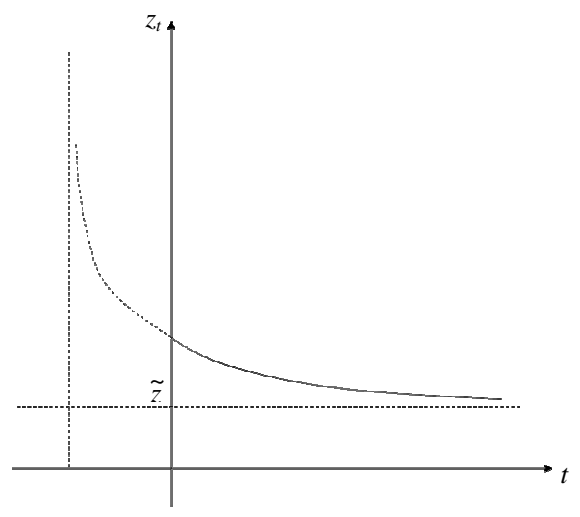

Figure 1. Evolution of dirty emissions

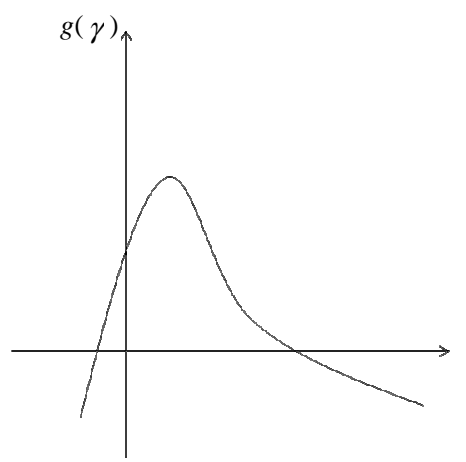

Figure 2. The BGP growth rate 
to a positive value of dirty emissions, unless we admit a stop in the economic development. This is consistent with the behavior of an advanced economy where, despite the presence of a high demand for environmental protection and a rise in technological innovations, it can be noted nonetheless a substitution amongst pollutants, whose pressure on the ecosystem is far away from disappearing.

This economy seems to mimic one where, to achieve balanced growth, pollution grows at the same level of output. However, we conclude that this economy behaves in a sustainable way only if natural capital grows more than technological sector. To summarize, it can be thought as a simple parable to explain why rich economies, despite their preferences towards clean air, and the presence of a technological sector that permits to substitute inputs in production, still achieve high levels of output, though associated with higher levels of emissions ( $\mathrm{CO}_{2}$, for example) to the atmosphere of the environment they live.

\subsection{The Reduced Model}

We can reduce the dimension of the canonical system given so far through the following convenient variable substitution

$$
\begin{aligned}
\frac{C}{K} & =x \\
\frac{Y}{K} & =m \\
\frac{C}{E} z^{\gamma} & =q
\end{aligned}
$$

and consequently end up with a new system in three dimensions, $x, m$, and $q$ :

$$
\begin{aligned}
& \dot{x}=\xi x-\left(\frac{1-\sigma}{\sigma}\right) m q+(\beta-1) m x+x^{2} \\
& \dot{m}=\eta m-\delta m^{2}+\frac{1}{\beta \sigma} m q \\
& \dot{q}=\xi q-\left(\frac{1-2 \sigma}{\sigma}\right) \frac{m q^{2}}{x}+(1+\gamma) q^{2}+\beta(1-\sigma) m q
\end{aligned}
$$

where

$$
\begin{aligned}
& \xi=\frac{(1-\sigma) \theta-\rho}{\sigma} \\
& \beta=\left(\frac{\gamma}{1+\gamma}\right)\left(\frac{1-\alpha}{\sigma}\right) \\
& \eta=\frac{\theta+\alpha \gamma(\varphi+\gamma)}{\gamma(1-\alpha)} \\
& \delta=\frac{1+\alpha \gamma}{1+\gamma}
\end{aligned}
$$

The associated Jacobian matrix at the steady state $\left(x^{*}\right.$, $\left.m^{*}, q^{*}\right)$ is then

\footnotetext{
${ }^{16}$ Since the eigenvalues of $J^{*}$ are the solutions of its characteristic equa-
} tion

$$
J^{*}=\left[\begin{array}{lll}
x^{*}+\left(\frac{1-\sigma}{\sigma}\right) \frac{m^{*} q^{*}}{x^{*}} & (\beta-1) x^{*}-\left(\frac{1-\sigma}{\sigma}\right) q^{*} & -\left(\frac{1-\sigma}{\sigma}\right) m^{*} \\
0 & -\delta m^{*} & \frac{1}{\beta \sigma} m^{*} \\
\left(\frac{1-2 \sigma}{\sigma}\right) \frac{m^{*} q^{* 2}}{x^{* 2}} & \beta(1-\sigma) q^{*}-\left(\frac{1-2 \sigma}{\sigma}\right) \frac{q^{* 2}}{x^{*}} & (1+\gamma) q^{*}-\left(\frac{1-2 \sigma}{\sigma}\right) \frac{m^{*} q^{*}}{x^{*}}
\end{array}\right]
$$

Studying the behavior of this economy while converging to the steady state needs particular attention, especially if we want to control for the presence of undesired outcomes due to the rise of indeterminacy problems. To this end, we apply the neat Routh-Hurwitz criterion to the structure of eigenvalues associated with $J^{*}$, and easily verify that $\operatorname{trJ} J^{*}>0$, and $\operatorname{DetJ}^{*}<0$. In this case, the sequence of signs becomes $(-,+, ?,-)$, the only possibility is thus two positive and one negative eigenvalues. The interior steady state is therefore determinate, or saddle path stable. ${ }^{16}$

This is quite a piece of news when dealing with a Romer-type economy, whose uniqueness of the equilibrium trajectory, largely studied in several papers, showed the need for some parameters of the model to belong to a particular defined set. For example, Asada et al. [17] study the stability properties of a social planner version of the Romer model and several modifications of it, including the complementarity of different intermediate goods introduced by Benhabib et al. [18], and find the emergence of Hopf bifurcation points and stable periodic solutions [19,20].

More recently, Slobodyan [9] reconsiders a slightly simpler version of Benhabib et al. [18], and derives the restrictions on the parameter values necessary to obtain an interior steady state solution. He shows that Hopf bifurcation leading from determinate steady state to a completely stable one does not exist, but that indeterminate steady state can become absolutely unstable (explosive) through Hopf bifurcation.

In this light, we ought to make a deep investigation, by relaxing the assumption made upon the research success parameter, $\gamma$, and show that in case we allow it to become negative, some indeterminacy problems may finally arise, and thus complicate the possibility to attain a sustainable equilibrium solution either. The next section is devoted to this end.

\subsection{A Numerical Analysis}

Without any loss of generality, and for the sake of simplicity, in this section we analyze a simpler version of the model set above, where we constrain $\sigma=1$. It is indeed like moving back to the $A H$ model, where the structure of preferences implies a logarithmic utility function.

Firstly, let us consider the case $\gamma>0$, then the Jacobian matrix easily reduces to

$$
J^{*}=\left[\begin{array}{lll}
x^{*} & -\delta x^{*} & 0 \\
0 & -\delta m^{*} & \frac{1}{\beta} m^{*} \\
-\frac{m^{*} q^{* 2}}{x^{* 2}} & \frac{q^{* 2}}{x^{*}} & \rho
\end{array}\right]
$$


with

$$
\begin{gathered}
\operatorname{trJ} J^{*}=2 \rho>0 \\
\operatorname{DetJ}^{*}=-\frac{\rho m^{*}}{\beta x^{*}}\left[q^{* 2}+\beta \delta x^{* 2}\right]<0
\end{gathered}
$$

the system is still characterized by a two-change of sign $(-,+, ?,-)$, which implies the local stability of the steady state solution.

In particular, stability of system $(S)$ needs

$$
\begin{aligned}
& x^{*}=\rho+\delta m^{*} \\
& \eta+\frac{1}{\beta} q^{*}=\delta m^{*} \\
& {\left[(1+\gamma) q^{*}-\rho\right] x^{*}+m^{*} q^{*}=0}
\end{aligned}
$$

which implies, solving for $m^{*}$, the following quadratic equation

$$
G(m)=a m^{* 2}-b m^{*}-c=0
$$

where

$$
\begin{aligned}
& a=\beta \delta[1-(\beta-1)(1+\gamma)]=\frac{\gamma(1-\alpha)(1+\alpha \gamma)(2+\alpha \gamma)}{(1+\gamma)^{2}} \\
& b=\frac{\left(\alpha \gamma^{2}+\theta\right)(2+\alpha \gamma)}{(1+\gamma)}+\frac{\rho(1+\alpha \gamma)}{1+\gamma}[1-\gamma(1-\alpha)] \\
& c=\rho\left(\rho+\theta+\alpha \gamma^{2}\right)
\end{aligned}
$$

and given $a>0$, and $c>0$, this allows us to understand why there is only one possible positive solution for $m^{*}$ in steady state, and the system is therefore locally stable, whatever the sign of $b$, as shown in Figure 3.

On the contrary, if we allow the research success parameter (i.e. the degree of pollution abatement), to fall below zero, $\gamma<0$, then some unexpected economic outcomes may arise. In particular, whenever $-\frac{1}{\alpha}<\gamma<-1$, we conclude that either $a, b<0$ or $c>0$, whose graphic representation in Figure 4 clearly shows the

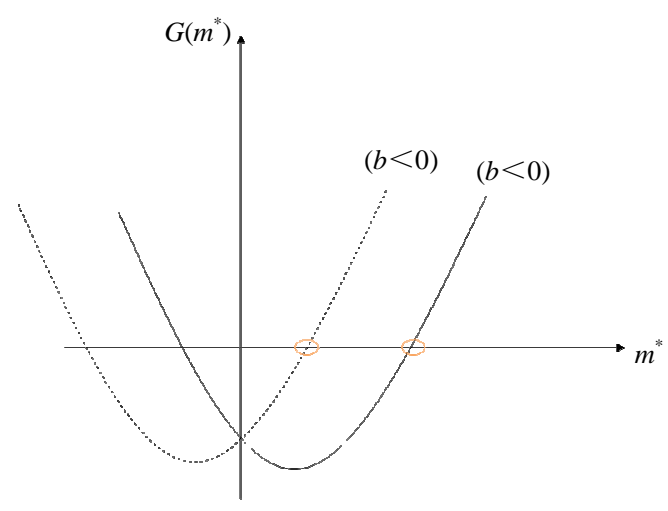

Figure 3. Unique equilibrium presence of two positive solutions for $G\left(m^{*}\right)=0$, and thus consequently signal the emergence of a multiplicity of equilibria.

To conclude, the new home-made inventories become a key indicator to achieve a long run sustainable equilibrium. On the one hand, in fact, we have shown so far that as long as an increase in the stock of knowledge is realized, i.e. $\gamma$ is positive, the economy converges to a saddle path stable steady state. On the other hand, when the home-made research sector experiences a decreasing level of new inventories, which means a negative value for $\gamma$, the economy is likely to manifest some indeterminacy problems. In this case, a multiplicity of equilibria is therefore possible to arise, and consequently generate a situation where the economy might be trapped in a lower equilibrium solution. Other non-economic factors are thus possibly acting as a means for equilibria to differ along the transition path towards the steady state.

\section{Concluding Remarks}

A clear connection between growth and the quality of the environment is complex. Some elements of environmental quality appear to improve with growth; others worsen; still others exhibit deterioration followed by amelioration. Despite this evidence, most studies dealing with the impact of environmental policy on growth ignore the adverse effect of pollution on productivity. The state of the environment may worsen with time if concentrations of pollutants accumulate or if consumer tastes shift towards pollution-intensive goods. The opposite does occur if technological innovations make abatement less costly or if increasing awareness causes an autonomous shift in public demands for environmental safeguards. To this end, only if technological progress has to provide the means to reducing the over-exploitation of natural resources, a sustainable growth can be possible.

To bridge the existing gap we set up a model close to Aghion and Howitt [6] and examine the problem of sustainable growth in presence of dirty (i.e. polluting)

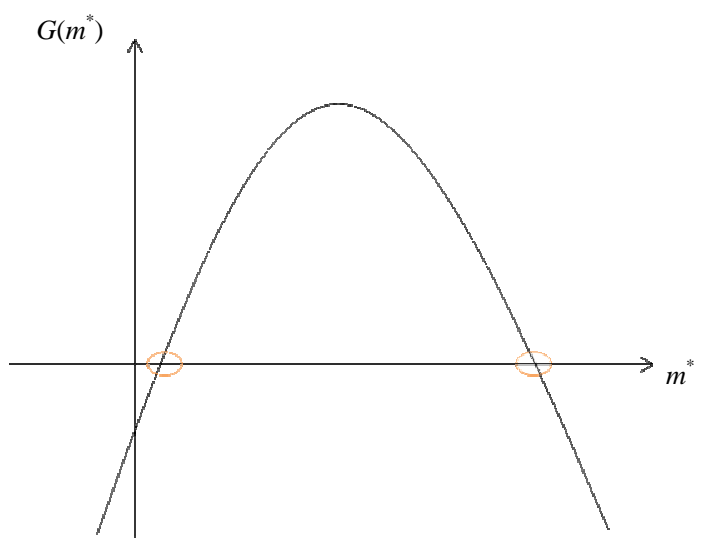

Figure 4. Multiple equilibria 
production processes. We show that under certain conditions a sustainable growth is always attainable. The main difference with respect to our analysis regards the definition of a non-separable utility function (where both consumption and the environment are seen as two substitutes), and a particular technological sector where both home-made and outsourcing research activities are considered.

Particular attention has been devoted to the transitional dynamics of the model around the steady state, where the role of home-made research has turned out as a key device for stability and uniqueness of equilibrium solutions. Indeed, if the home-made research parameter is allowed to be negative, some indeterminacy problems arise, and multiple equilibria are likely to emerge. In this latter case, some non-economic factors become crucial in the solution of our decision making problem. This is consistent to how real economies nowadays behave, whenever their different cultural backgrounds impinge on the approach used to tackle the problem of a sustainable allocation of the available natural resources amongst generations. That is also likely to influence the development path of these economies towards the steady state, and eventually trap the systems into an unavoidable low equilibrium level.

\section{REFERENCES}

[1] D. I. Stern, "The rise and fall of the environmental kuznets curve," World Development 32, pp. 1419-1439, 2004.

[2] S. Smulders, "Environmental policy and sustainable economic growth: An endogenous growth perspective," De Economist 143, pp. 163-195, 1995.

[3] A. L. Bovenberg and S. Smulders, "Environmental quality and pollution-augmenting technological change in a two-sector endogenous growth model," Journal of Public Economics 57, pp. 369-391, 1995.

[4] A. L. Bovenberg and S. Smulders, "Transitional impacts of environmental policy in an endogenous growth model," International Economic Review 37, pp. 861-893, 1996.

[5] K. Pittel, "Sustainability and endogenous growth," Edward Elgar, Cheltenham, 2003.

[6] P. Aghion and P. Howitt, "Endogenous growth theory (2nd edition)," MIT Press, Cambridge, Massachusetts, 1998.
[7] A. Grimaud and F. Ricci, "The growth-environment trade-off: horizontal vs vertical innovations," Fondazione ENI Enrico Mattei Working Paper n, pp. 34-99, 1999.

[8] P. Schou, "Polluting non renewable resources and growth," Environmental and Resource Economics 16 (2), pp. 211-227, 2000.

[9] S. Slobodyan, "Indeterminacy and stability in a modified Romer model," Journal of Macroeconomics 29, pp. 169-177, 2007.

[10] A. Grimaud, "Pollution permits and sustainable growth in a schumpeterian model," Journal of Environmental Economics and Management 38, pp. 249-266, 1999.

[11] S. I. Restrepo-Ochoa and J. Vazquez, "Cyclical features of the Uzawa--Lucas endogenous growth model," Economic Modelling 21, pp. 285-322, 2004.

[12] M. A. Gomez, "Transitional dynamics in an endogenous growth model with physical capital," Human Capital and R\&D. Studies in Nonlinear Dynamics \& Econometrics 9 (1), article 5, 2005.

[13] S. Smulders, "Economic growth and environmental quality," In: Folmer, H., Gabel, L. (Eds), Principles of Environmental and Resource Economics, Edward Elgar, Chapter 20, pp. 602-664, 2000.

[14] S. Smulders, "Entropy, environment, and endogenous economic growth," International Tax and Public Finance 2, pp. 319-340, 1995.

[15] I. Musu, "Transitional dynamics to optimal sustainable growth," Fondazione ENI Enrico Mattei Working Paper n. 50.95, 1995.

[16] R. J. Barro and X. Salai-Martin, "Economic growth," McGraw-Hill, New York, 1995.

[17] T. Asada, W. Semmler, and A. J. Novak, "Endogenous growth and the balanced growth equilibrium," Research in Economics 52, pp. 189-212, 1998.

[18] J. Benhabib, R. Perli, and D. Xie, Monopolistic competition, indeterminacy and growth. Ricerche Economiche 48, pp. 279-298, 1994.

[19] L. G. Arnold, "Endogenous technological change: A note on stability," Economic Theory 16, pp. 219-226, 2000.

[20] L. G. Arnold, "Stability of the market equilibrium in Romer's model of endogenous technological change: A complete characterization," Journal of Macroeconomics 22, pp. 69-84, 2000.

(Edited by Vivian and Ann) 


\section{Appendix A}

The current value Hamiltonian for the maximization problem is given by

$$
\begin{aligned}
& H_{c}=\frac{(C E)^{1-\sigma}-1}{1-\sigma}+\lambda\left[A^{\alpha}\left(1-S_{A}\right)^{\alpha} K^{1-\alpha} z-C\right]+ \\
& +\mu\left[\theta E-A^{\alpha}\left(1-S_{A}\right)^{\alpha} K^{1-\alpha} z^{1+\gamma}\right]+\vartheta\left[\left(\varphi+\gamma S_{A}\right) A\right]
\end{aligned}
$$

where $\lambda, \mu$ and $\vartheta$ denote the costate variables associated with the accumulation of physical capital, natural capital and knowledge capital, respectively.

First order conditions can be written as:

1.a $\quad\left[\frac{\partial H_{c}}{\partial C}=0\right]$ :

$$
\frac{\partial H_{c}}{\partial C}=C^{-\sigma} E^{1-\sigma}-\lambda=0 \Rightarrow C^{-\sigma} E^{1-\sigma}=\lambda
$$

1.b $\quad\left[\frac{\partial H_{C}}{\partial z}=0\right]:$

$\frac{\partial H_{c}}{\partial z}=\lambda A^{\alpha}\left(1-S_{A}\right)^{\alpha} K^{1-\alpha}-\mu(1+\gamma) A^{\alpha}\left(1-S_{A}\right)^{\alpha} K^{1-\alpha} z^{\gamma}=0$

that is simply

$$
\lambda=\mu(1+\gamma) z^{\gamma}
$$

$$
\text { 1.c } \quad\left\lfloor\frac{\partial H_{c}}{\partial S_{A}}=0\right\rfloor \text { : }
$$

$$
\begin{gathered}
\frac{\partial H_{c}}{\partial S_{A}}=-\lambda \alpha A^{\alpha}\left(1-S_{A}\right)^{\alpha-1} K^{1-\alpha} z+\mu \alpha A^{\alpha}\left(1-S_{A}\right)^{\alpha-1} \\
K^{1-\alpha} z^{1+\gamma}+\vartheta \gamma A=0
\end{gathered}
$$

or rather, using (A.3a) it becomes

$$
\mu \gamma \alpha A^{\alpha}\left(1-S_{A}\right)^{\alpha-1} K^{1-\alpha} z^{1+\gamma}=\vartheta A
$$

Equation of motion for each costate variable is given by

$$
\begin{aligned}
& \dot{\lambda}=-\frac{\partial H_{c}}{\partial K}+\lambda \rho \\
& \dot{\mu}=-\frac{\partial H_{c}}{\partial E}+\mu \rho \\
& \dot{\vartheta}=-\frac{\partial H_{c}}{\partial A}+\vartheta \rho
\end{aligned}
$$

and we can simply derive, by means of the conditions obtained above:

$$
\begin{aligned}
& \frac{\dot{\lambda}}{\lambda}=-\left(\frac{\gamma}{1+\gamma}\right)(1-\alpha) A^{\alpha}\left(1-S_{A}\right)^{\alpha} K^{-\alpha} z+\rho \\
& \frac{\dot{\mu}}{\mu}=-\frac{C^{1-\sigma} E^{-\sigma}}{\mu}-\theta+\rho \\
& \frac{\dot{\vartheta}}{\vartheta}=-\frac{\mu}{\vartheta} \gamma \alpha A^{\alpha-1}\left(1-S_{A}\right)^{\alpha} K^{1-\alpha} z^{1+\gamma}-\gamma S_{A}+\rho
\end{aligned}
$$

by substituting out condition (A.4a) into the law of motion of $\vartheta$, it follows

$$
\frac{\dot{\vartheta}}{\vartheta}=\rho-(\varphi+\gamma)
$$

whereas, taking logs in (A.2) and differentiating, we have

$$
\frac{\dot{\lambda}}{\lambda}=-\sigma \frac{\dot{C}}{C}+(1-\sigma) \frac{\dot{E}}{E}
$$

From condition (A.6) derives

$$
\dot{\mu}=-C^{1-\sigma} E^{-\sigma}-\mu \theta+\mu \rho
$$

or, alternatively,

$$
\frac{\dot{\mu}}{\mu}=-\frac{U_{E}}{\mu}-\theta+\rho
$$

given that $\frac{\partial U(\cdot)}{\partial E}=C^{1-\sigma} E^{-\sigma}=U_{E}$. But substituting out $\mu$ in the RHS, by means of (A.3a), we obtain

$$
\frac{\dot{\mu}}{\mu}=-\frac{U_{E}}{\lambda}(1+\gamma) z^{\gamma}-\theta+\rho
$$

Since $\lambda=U_{C}$, from FOC, we have

$$
\frac{\dot{\mu}}{\mu}=-\frac{U_{E}}{U_{C}}(1+\gamma) z^{\gamma}-\theta+\rho
$$

and finally, since equilibrium requires that $\frac{U_{E}}{U_{C}}=\frac{C}{E}$, it follows

$$
\frac{\dot{\mu}}{\mu}=\rho-\theta-\frac{C}{E}(1+\gamma) z^{\gamma}
$$

- Arrow sufficiency theorem holds since the maximized Hamiltonian, evaluated along the optimal control variables, is concave in all the state variables, as we can simply check through the sing of the minors of the Hessian matrix, whose determinant implies the following sings

$$
|H|=\left|\begin{array}{ccc}
- & 0 & + \\
0 & - & 0 \\
+ & 0 & -
\end{array}\right|
$$

- hence, $\left|H_{1}\right|<0,\left|H_{2}\right|>0$, and $\left|H_{3}\right|<0$ iff $A>1$, that is the number of designs must necessarily be greater that one.

- Transversality conditions for a free terminal state hold for all shadow prices, and are given by

$$
\begin{aligned}
& \lim _{t \rightarrow \infty} \lambda K e^{-\rho t}=\tilde{\lambda} e^{(1-2 \sigma) g t} \tilde{K} e^{g t} e^{-\rho t}=\tilde{\lambda} \tilde{K} e^{-(2 \sigma g+\rho) t}=0 \\
& \lim _{t \rightarrow \infty} \mu E e^{-\rho t}=\tilde{\mu} e^{(1-2 \sigma) g t} \tilde{E} e^{g t} e^{-\rho t}=\tilde{\mu} \tilde{E} e^{-(2 \sigma g+\rho) t}=0 \\
& \lim _{t \rightarrow \infty} \vartheta A e^{-\rho t}=\tilde{\vartheta} e^{(\rho-\varphi-\gamma) t} \tilde{A} e^{g t} e^{-\rho t}=\tilde{\vartheta} \tilde{A} e^{(g-\varphi-\gamma) t}=0
\end{aligned}
$$

- Where $\tilde{\lambda}, \tilde{\mu}, \tilde{\vartheta}$, and $\tilde{K}, \tilde{E}, \tilde{A}$, are the shadow prices and the state-values on the balanced growth path; 
- Moreover, for free time $t$, we need to show that $\lim _{t \rightarrow \infty} H=0$, which is always verified due to convergence towards zero of both the discounted utility function, $\lim _{t \rightarrow \infty} U(\cdot) e^{-\rho t}=0$, and all the multipliers, as proved above.

Transitional dynamics of the problem can be studied by applying the Routh-Hurwitz criterion to the autonomous system

$$
\begin{gathered}
\dot{x}=\xi x-\left(\frac{1-\sigma}{\sigma}\right) m q+(\beta-1) m x+x^{2} \\
\dot{m}=\eta m-\delta m^{2}+\frac{1}{\beta \sigma} m q \\
\dot{q}=\xi q-\left(\frac{1-2 \sigma}{\sigma}\right) \frac{m q^{2}}{x}+(1+\gamma) q^{2}+\beta(1-\sigma) m q
\end{gathered}
$$

and the associated Jacobian matrix, evaluated along the steady state

$$
\begin{aligned}
& J^{*}=\left[\begin{array}{lll}
J_{11}^{*} & J_{12}^{*} & J_{13}^{*} \\
J_{21}^{*} & J_{22}^{*} & J_{23}^{*} \\
J_{31}^{*} & J_{32}^{*} & J_{33}^{*}
\end{array}\right] \\
& \operatorname{DetJ}^{*}=\left[x^{*}+\left(\frac{1-\sigma}{\sigma}\right) \frac{m^{*} q^{*}}{x^{*}}\right]\left\{-\delta(1+\gamma) m^{*} q^{*}+\delta\left(\frac{1-2 \sigma}{\sigma}\right) \frac{m^{* 2} q^{*}}{x^{*}}-\left(\frac{1-\sigma}{\sigma}\right) m^{*} q^{*}+\left(\frac{1-2 \sigma}{\beta \sigma^{2}}\right) \frac{m^{*} q^{* 2}}{x^{*}}\right\}+ \\
& \left(\frac{1-2 \sigma}{\sigma}\right) \frac{m^{*} q^{* 2}}{x^{* 2}}\left[\left(\frac{\beta-1}{\beta \sigma}\right) m^{*} x^{*}-\left(\frac{1-\sigma}{\beta \sigma^{2}}\right) m^{*} q^{*}-\delta\left(\frac{1-\sigma}{\sigma}\right) m^{* 2}\right]<0
\end{aligned}
$$

which implies also

$$
\begin{gathered}
\left(\frac{1-2 \sigma}{\sigma}\right) \frac{m^{*} q^{* 2}}{x^{* 2}}\left[\left(\frac{\beta-1}{\beta \sigma}\right) m^{*} x^{*}-\left(\frac{1-\sigma}{\beta \sigma^{2}}\right) m^{*} q^{*}-\delta\left(\frac{1-\sigma}{\sigma}\right) m^{* 2}\right]< \\
{\left[q^{*}+\left(\frac{1-\sigma}{\sigma}\right) \frac{m^{*} q^{* 2}}{x^{* 2}}\right]\left\{\left[\delta(1+\gamma)+\left(\frac{1-\sigma}{\sigma}\right)\right] m^{*} x^{*}-\left(\frac{1-2 \sigma}{\beta \sigma^{2}}\right) m^{*} q^{*}-\delta\left(\frac{1-2 \sigma}{\sigma}\right) m^{* 2}\right\}}
\end{gathered}
$$

since it is always verifiable that

$$
\begin{gathered}
\frac{1-\sigma}{\sigma}>\frac{1-2 \sigma}{\sigma} \\
\frac{\beta-1}{\beta \sigma}<\delta(1+\gamma)+\left(\frac{1-\sigma}{\sigma}\right)
\end{gathered}
$$

or rather

$$
\left[\alpha(1-\alpha) \gamma^{2}+(1+\gamma)\right] \sigma>0
$$

